\title{
Expression of Concern
}

\section{Adrenergic-mediated increases in $I N H B A$ drive CAF phenotype and collagens}

Archana S. Nagaraja, Robert L. Dood, Guillermo Armaiz-Pena, Yu Kang, Sherry Y. Wu, Julie K. Allen, Nicholas B. Jennings, Lingegowda S. Mangala, Sunila Pradeep, Yasmin Lyons, Monika Haemmerle, Kshipra M. Gharpure, Nouara C. Sadaoui, Cristian Rodriguez-Aguayo, Cristina Ivan, Ying Wang, Keith Baggerly, Prahlad Ram, Gabriel Lopez-Berestein, Jinsong Liu, Samuel C. Mok, Lorenzo Cohen, Susan K. Lutgendorf, Steve W. Cole, and Anil K. Sood

Original citation: JCI Insight. 2017;2(16):1-17. https://doi.org/10.1172/jci.insight.93076.

Citation for this expression of concern: JCI Insight. 2018;3(11):e122389. https://doi.org/10.1172/jci. insight.122389.

In Figure 5E, the siControl Stress and siNHBA Control samples appear to be the same. The Editorial Board is pursuing further investigation of this matter, and we will inform our readers of the outcome when the investigation is complete. 